\title{
Theoretische Vereinheitlichung der Physik im Lichte der Geschichte der Wissenschaften
}

\author{
Von Prof. Dr. Emil J. Walter
}

Wer versucht, sich einige möglicherweise als gültig erweisende Gedanken über die künftige Entwicklung der wissenschaftlichen Forschung und ihre Grenzen zu machen, greift in seinen Überlegungen zweckmäßig auf die historische Entwicklung der betreffenden Disziplin zurück. Versuchen wir daher, aus der faktischen Entwicklung der theoretischen und experimentellen Physik von der Antike bis zur Gegenwart hypothetische Schlüsse in bezug auf die künftigen Möglichkeiten der physikalischen Grundlagenforschung zu ziehen.

Wissenschaftliches Denken hat im Altertum zunächst im griechischen Kleinasien, bei den ionischen Naturphilosophen, Fuß gefaßt. Diese an und für sich erstaunliche Tatsache dürfte auf die Erfindung der Buchstabenschrift durch das Handelsvolk der Griechen zurückzuführen sein. Die Buchstabenschrift erleichtert im Gegensatz zur Bilderschrift das abstrakte Denken. Es kann kein historischer Zufall sein, daß, wie bereits G. Sarton in seiner Introduction to the History of Science gezeigt hat, sowohl die indische wie auch die chinesische Kultur nur in ganz beschränktem Maße den Übergang vom vorwissenschaftlichen zum Wissenschaftlichen Denken gefunden haben.

Bei den Griechen wurde zunächst unter dem Begriff der Philosophie, d.h. der Liebe zur Weisheit, bzw. «zum Wort als dem Träger geistiger Inhalte» all das zusammengefaßt, was durch den neuen Denkstil erfaßt werden konnte, vor allem die grundlegenden Prinzipien, welche das Weltgeschehen bestimmen sollten. Auf die Periode der ionischen Naturphilosophie folgte die Periode der geistig zusammenfassenden Synthese, welche in der antiken Lehre der Urelemente, der Luft, des Wassers, der Erde und des Feuers, gipfelte. Philosophie war schon im Altertum Weltanschauungslehre der Gebildeten im Gegensatz zur volkstümlichen religiösen Welt der Götter.

Es ist wenig sinnvoll, in diesem Zusammenhang auf allgemein bekannte Fakten der Philosophiegeschichte zurückzugreifen, auf den idealistischen Grundzug der Philosophie Platons im Gegensatz zum stärker betonten Empirismus seines bedeutendsten Schülers Aristoteles oder hinzuweisen auf Einzelleistungen der Gruppe der Mathematiker um Euklid, des Archimedes, des Hipparch oder vieler anderer bedeutender Forscher des Altertums. In einem langsamen, sich über Jahrhunderte hinziehenden Prozeß führte die Beschäftigung mit philosophischen Problemen schon im griechischen Altertum und im Hellenismus zur Ablösung 
einzelner wissenschaftlicher Disziplinen aus dem Gesamtbereich dessen, was zunächst Philosophie im weitesten Sinne des Wortes war. In erster Linie verselbständigten sich Mathematik, Logik, Astronomie, Zoologie und die Mechanik.

Jene Wissensgebiete, die heute als physikalische bezeichnet werden, lösten sich mehr oder weniger deutlich schon im griechischen Altertum insofern von der philosophischen Spekulation ab, sobald konkrete Fakten im einzelnen genauer untersucht wurden. Dabei gingen die entsprechenden Untersuchungen ganz unzweideutig von den einzelnen Sinnesbereichen des Menschen aus. Die durch Tastund Gesichtssinn erfaßbare Körperwelt führte zur Mechanik, während Erscheinungen der Reflexion, durch den Gesichtssinn erkennbar, Veranlassung gaben, eine Optik aufzubauen. Analog kann von Ansätzen zu einer Wärmelehre bzw. Kalorik ausgehend vom Temperatursinn und von der Akustik ausgehend vom Gehörsinn gesprochen werden. Dabei ist es kennzeichnend, daß diese vier Teilgebiete physikalischen Denkens sich im wesentlichen auf die Beschreibung der Vorgänge beschränkten. Selbst die atomistischen Deutungsversuche eines Leukipp und Demokrit der Körperwelt beschränkten sich auf mechanistische Vorstellungen. Zur Erfassung physikalischer Kräfte im modernen Sinne vermochte das antike Denken nicht vorzustoßen. Wie in der Mystik oder den Religionen bediente man sich vorwissenschaftlicher Begriffe soziomorpher, technomorpher, biomorpher oder psychomorpher Vorstellungen oder Analogien, um Naturerscheinungen verständlich zu machen. Eine Dynamik oder nicht anthropomorphe Kräftelehre gab es im Altertum nicht, weil vor der Erfindung der Feuerwaffen kein Bedürfnis nach einer Bewegungslehre bzw. Dynamik bestand. In dem berühmten Lehrgedicht De rerum naturae des Lucretius Carus (96-56 v. Chr.) ist zwar die materialische Lehre der Atome mitsamt dem weltanschaulichen System Epikurs übernommen worden, aber von einer wissenschaftlichen, die Stufe der Beschreibung überschreitenden Erklärung der Naturvorgänge ist noch keine Rede.

Diese Situation sollte sich bis ins 17. Jahrhundert nicht ändern. Das Bedürfnis nach Ordnung des Kalenders erzwang die Revision der überlieferten astronomischen Vorstellungen durch Kopernikus. Die Technik hatte in der Zwischenzeit bedeutende Fortschritte gemacht. Außer dem Schwarzpulver, das die Erfindung der Feuerwaffen auslöste, übernahmen die Europäer von den Chinesen den Kompaß. Dieser erleichterte die Hochseeschiffahrt und damit die Entdeckung Amerikas. Die Feuerwaffen begründeten die Herrschaft der Weißen über die farbigen Völker. Erfindung der Buchdruckerkunst, technische Entwicklung von Baukunst, Schiffsbau, Waffenlehre und die Erfindung von Fernrohr und Lupe förderten das Verständnis für experimentelle Beobachtungen.

Die Folgen stellten sich im 17. Jahrhundert dank den geistigen Leistungen einzelner, selbständig denkender Forscher ein. Im Jahre 1600 veröffentlichte William Gilbert sein klassisches Werk De magnete und begründete damit Magnetik und Elektrik als neue physikalische Disziplinen. Er führte den Begriff «vis electri- 
ca», d.h. «Bernsteinkraft» in die Wissenschaft ein. Die Untersuchung der Feldkräfte des magnetischen und des elektrischen Feldes, wofür wir im Gegensatz zu Fischen und wohl auch Vögeln über kein besonders Sinnesorgan verfügen, hatte begonnen.

1633 erschienen die Discorsi, das erste moderne Lehrbuch der Physik, zwei neue Wissenschaften, die Mechanik und die Fallgesetze betreffend, von Galileo Galilei, nachdem bereits 1610 und 1618 Johannes Kepler die drei Planetengesetze errechnet hatte. In der Folge häuften sich die grundsätzlichen Leistungen der Naturforscher. Es würde zu weit führen, auf allgemein bekannte Tatsachen hinzuweisen. Den absoluten Höhepunkt dieser Entwicklung einer Synthese der irdischen Beobachtung der Fallgesetze, der Formulierung des Trägheitsgesetzes mit astronomischer Forschung bildete 1687 die Veröffentlichung der Principia mathematica durch Isaac Newton. In diesem Werke formulierte Newton die Gesetze der klassischen Mechanik und leitete damit eine neue Phase der wissenschaftlichen Entwicklung ein. Noch standen Mechanik, Akustik, Kalorik, Optik, Magnetik und Chemie als scheinbar unabhängige Einzeldisziplinen nebeneinander. Aber es mehrten sich die Versuche, allgemein die Naturerscheinungen nach einheitlichen Gesichtspunkten zu erklären, z. B. die Wärme als dynamisches Problem oder als Wirkung eines besonderen Wärmestoffes zu verstehen, die elektrischen und magnetischen Erscheinungen entweder als Wirkungen besonderer Substanzen oder mechanistisch als Zustände eines hypothetischen Weltäthers zu deuten.

Bis zum Beginn des 20. Jahrhunderts, dem Zeitalter der zweiten industriellen Revolution und der Verwissenschaftlichung von Technik und Wirtschaft, entwickelten sich vom theoretischen Standpunkte aus gesehen und geführt durch das physikalische Experiment die Physik zur klassischen Mechanik und Thermodynamik einerseits und zur elektromagnetischen Theorie des Lichtes andererseits. Um 1800 entschied Rumford in München durch klassische Versuche die Frage, ob die Wärmeerscheinungen substantiell oder dynamisch zu erklären seien. Die entsprechende dynamische Theorie wurde im Zusammenhang mit den durch die Ausbreitung der Dampfmaschinen in der Mitte des 19. Jahrhunderts geförderte Entdekkung des Erhaltungssatzes der Energie durch Robert Mayer und Heinrich Helmholtz als kinetische Theorie der Wärme durch E.Clausius und W.Thomson, den späteren Lord Kelvin geschaffen. Damit waren die Himmelsmechanik, die Mechanik der festen, flüssigen und gasförmigen Körper, die Wärmelehre und die Akustik zu einem einheitlichen Lehrgebäude zusammengefaßt worden. Die Körperwelt wurde aufgebaut gedacht aus Atomen und Molekülen, einer Art von Korpuskeln und ihre gegenseitige Einwirkung auf Adhäsion, Kohäsion, chemische Anziehungskräfte, Stoßwirkungen und Gravitation zurückgeführt.

Ungefähr zur gleichen Zeit, in den Jahren 1843 bis 1846 gelang es Michael Faraday zu zeigen, daß die Ebene eines polarisierten Lichtstrahles durch die 
Einschaltung eines Magnetfeldes unter bestimmten Bedingungen gedreht werden kann. Damit war die Grundlage gegeben für die Schaffung der elektromagnetischen Lichttheorie durch Maxwell Ende der sechziger Jahre. Seit Beginn des 19. Jahrhunderts hatten Oersted, Ampère, Faraday u. a. Forscher den engen Zusammenhang von Magnetismus und elektrischen Strömen experimentell erforscht. Nach den Vorstellungen Faradays wurden magnetische und elektrische Kraftfelder und im Gegensatz zu der klassischen Mechanik Nahwirkungskräfte mit endlicher Ausbreitungsgeschwindigkeit, nämlich der Lichtgeschwindigkeit angenommen.

Wesentlich ist, daß die theoretische Zusammenfassung der physikalischen Forschung in zwei Teilgebiete, die Thermodynamik einerseits und die elektromagnetische Strahlentheorie andererseits, überhöht durch die Postulate der Erhaltung der Energie und der Masse, eine zwingende theoretische Konsequenz langer experimenteller Versuchsreihen war. Dieser theoretische Vereinheitlichungsprozeß der Physik setzte sich - wie allgemein bekannt ist - im 20. Jahrhundert fort. Durch die im Jahre 1900 erstmals durch Max Planck formulierte, aus der Beschäftigung mit dem Problem der Umwandlung von Wärmeenergie in Licht- bzw. Strahlungsenergie entstandene Quantentheorie und der durch Albert Einstein 1905 formulierten Lichtquantenhypothese und der im gleichen Jahre unter dem Titel Zur Elektrodynamik bewegter Körper von Albert Einstein aufgestellten speziellen Relativitätstheorie entstand über die Deutung des Baues der Atome durch Rutherford, Bohr, Heisenberg und viele andere Forscher in den dreißiger Jahren die moderne, allgemeine relativistische Quantenmechanik der Materie. Damit ist in der Mitte des gegenwärtigen Jahrhunderts der theoretische Vereinheitlichungsprozeß der Physik mitsamt der Chemie als Atomphysik prinzipiell abgeschlossen worden.

Vom theoretischen Standpunkte aus ist keine weitere Vereinheitlichung der Physik mehr möglich. Mechanik, Akustik, Kalorik, Optik, Magnetik, Elektrik und Chemie sind heute, vom grundsätzlichen Standpunkte aus gesehen, nur noch Teile der umfassenden Naturwissenschaft, der Physik. Selbstverständlich ist unser Wissen seit dem Altertum ungeheuer gewachsen und müssen in den verschiedenen Teilgebieten der Physik jeweilen dem Forschungsobjekt entsprechende besondere Methoden angewendet werden. Aber offenbar ist damit - im großen gesehen - eine grundsätzliche Grenze der Grundlagenforschung erreicht. Die Welt der Physik zerfällt in den Bereich der elektromagnetischen Strahlung von den Langwellen bis zu den Gammastrahlen und den Bereich der Korpuskeln von den Bausteinen der Atome und Moleküle bis zu den sogenannten Elementarteilchen. Das periodische System der Elemente, ein theoretisches Rätsel zu Beginn dieses Jahrhunderts, hat seine theoretische Deutung gefunden und dient heute zur bewußten Erzeugung von transuranen Elementen. Das System der Elementarteilchen birgt noch zahlreiche Rätsel, deren Deutung in engster Verknüpfung mit den 
Fortschritten der Hochenergiephysik und der Astrophysik zu suchen sein dürfte. Offenbar bestimmen vier Kraftfelder das Geschehen der physikalischen wie auch der biologischen Welt, das Gravitationsfeld die Bewegungen im Kosmos, die elektromagnetischen Felder zwischen Atomen und Molekülen die chemischen Vorgänge, die Felder der schwachen und starken Wechselwirkung die Vorgänge im Innern der Atomkerne und die Prozesse der Wechselwirkung zwischen Strahlung und den Elementarteilchen. Zwischen diesen vier Kraftfeldern breiten sich die Wirkungen mit Lichtgeschwindigkeit aus, wodurch das alte Problem des Determinismus im Sinne der Minkowski-Welt eine moderne Lösung erfährt, da ja in jedem Weltpunkt $x, y, z$ und $t$ sich der Wirkungskegel der Vergangenheit und die Wirkung in die Zukunft überschneiden, die kausale Implikation in der Physik nicht mehr im Sinne des Weltbildes von Laplace aufgefaßt werden kann.

Ist die Schlußfolgerung zu kühn, wenn wir festhalten, aus theoretischen Gründen kann die Physik in dem Sinne keine grundsätzlich neuen Entdeckungen mehr machen, weil ihre theoretische Vereinheitlichung dies ausschließt? Zweifellos wird es gelingen, zu den bisherigen Kenntnissen noch viele zusätzliche Erkenntnisse zu gewinnen, aber Durchbrüche, wie sie bis zu den dreißiger Jahren dieses Jahrhunderts gelungen sind, sind nur noch auf dem Gebiete der Elementarteilchenphysik zu erwarten. Es scheint, daß neben dem absoluten Nullpunkt der Temperatur auch ein soluter Siedepunkt der Materie, wie dies an der Einsiedler Tagung der SNG dargestellt wurde, nachgewiesen werden kann. Zwangsläufig dürfte sich dabei künftig das Interesse der naturwissenschaftlichen Forschung von der physikalischen Grundlagenforschung der biologischen Grundlagenforschung und der Erforschung der sozialen Prozesse zuwenden, damit die Menschheit nicht von den irrationalen Folgen des technischen Fortschrittes überrannt und dem kosmischen Tod überliefert wird. Ganzheitliche Betrachtung der Entwicklung der Physik im Lichte ihrer Geschichte zwingt zur Besinnung auf künftige Möglichkeiten und notwendige Aufgaben, wenn die gesamte Menschheit ihre künftige Existenz auf der Erde, diesem «blauen» Planeten nicht gefährden will. 